\title{
Finite type annular ends for harmonic functions
}

\author{
William H. Meeks III* Joaquín Pérez ${ }^{\dagger}$
}

July 7, 2021

\begin{abstract}
In this paper we describe the notion of an annular end of a Riemann surface being of finite type with respect to some harmonic function and prove some theoretical results relating the conformal structure of such an annular end to the level sets of the harmonic function. As an application of these results, we obtain important information on the conformal type of any properly immersed minimal surface $M$ in $\mathbb{R}^{3}$ with compact boundary and which intersects some plane transversely in a finite number of arcs; in particular, such an $M$ is a parabolic Riemann surface. This information is applied by the authors in [5] to classify the asymptotic behavior of annular ends of a complete embedded minimal annulus with compact boundary in terms of the flux vector along its boundary. In the present paper, we apply this information to understand and characterize properly immersed minimal surfaces in $\mathbb{R}^{3}$ of finite total curvature, in terms of their intersections with two nonparallel planes.
\end{abstract}

Mathematics Subject Classification: Primary 53A10, Secondary 49Q05, 53C42 Key words and phrases: Minimal surface, finite type, harmonic function, parabolic Riemann surface, hyperbolic Riemann surface, angular limits.

\section{Introduction.}

Given a nonconstant harmonic function $f: M \rightarrow \mathbb{R}$ on a noncompact Riemann surface with compact boundary, we say that an annular end ${ }^{1} E$ of $M$ has finite type for $f$ if for some $t_{0} \in \mathbb{R}$, the one-complex $f^{-1}\left(t_{0}\right) \cap E$ has a finite number of ends.

\footnotetext{
${ }^{*}$ This material is based upon work for the NSF under Award No. DMS-1309236. Any opinions, findings, and conclusions or recommendations expressed in this publication are those of the authors and do not necessarily reflect the views of the NSF.

${ }^{\dagger}$ Research supported in part by the MINECO/FEDER grant no. MTM2014-52368-P.

${ }^{1}$ A proper subdomain $E \subset M$ is an annular end if it is homeomorphic to $\mathbb{S}^{1} \times[0,1)$.
} 


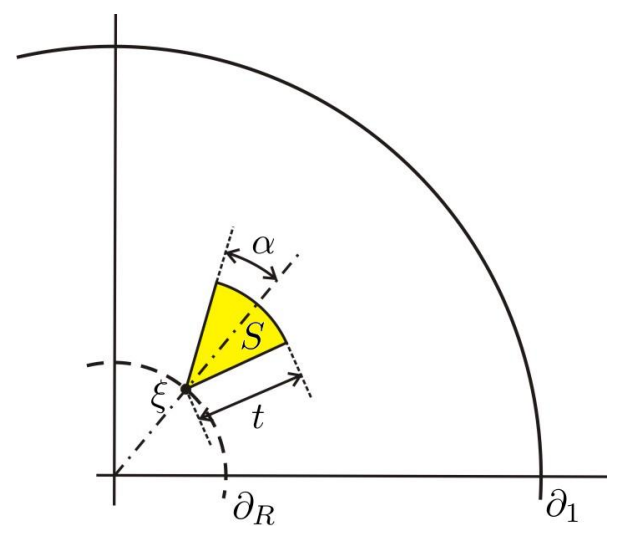

Figure 1: Angular sector in $A(R, 1)$, centered at $\xi \in \partial_{R}$.

Observe that if $E^{\prime}$ is an annular subend of $E$, then $E^{\prime}$ has finite type for $f$ if and only if $E$ has finite type for $f$. Thus, in the sequel we will assume that $f$ has no critical points in the boundary of $E$.

Note that $f^{-1}\left(t_{0}\right)$ might fail to be smooth: at each critical point $p$ of $f$ lying in $f^{-1}\left(t_{0}\right)$, this level set consists locally of an equiangular system of curves crossing at $p$; also note that $f^{-1}\left(t_{0}\right)$ cannot bound a compact subdomain in $E-\partial E$ by the maximum principle. These observations imply that $f^{-1}\left(t_{0}\right) \cap E$ has a finite number of ends if and only if $f^{-1}\left(t_{0}\right) \cap E$ has a finite number of components and a finite number of crossing points.

When $E$ is an annular end of finite type for $f$, we will prove several results on the level sets of $\left.f\right|_{E}$ depending on the conformal type of $E$. In order to describe these results, we first fix some notation. For $R \in[0,1)$, let $A(R, 1)=\{z \in \mathbb{C} \mid$ $R<|z| \leq 1\}, \bar{A}(R, 1)=\{z \in \mathbb{C}|R \leq| z \mid \leq 1\}, \partial_{R}=\{t \in \mathbb{C}|| z \mid=R\}$ and $\partial_{1}=\{z \in \mathbb{C}|| z \mid=1\}$. Thus, the closure $\bar{A}(0,1)$ in $\mathbb{C}$ is the closed unit disk $\overline{\mathbb{D}}$ and $A(0,1)=\overline{\mathbb{D}}-\{0\}$. If $0<R<1$ and $F: A(R, 1) \rightarrow \mathbb{C}$ is a holomorphic function, then $F$ is said to have angular limit $F(\xi) \in \mathbb{C} \cup\{\infty\}$ at $\xi \in \partial_{R}$ if $\lim _{z \rightarrow \xi, z \in S} F(z)$ exists and equals $F(\xi)$ for every angular sector $S \subset A(R, 1)$ centered at $\xi$, whose median line is the radial arc $[1,2] \xi$, with small radius $t \in(0,1-R)$ and total angle $2 \alpha, 0<\alpha<\frac{\pi}{2}$, see Figure 1 . This definition of having angular limit can be directly extended to (real valued) harmonic functions.

We can now state our main result.

Theorem 1.1 Suppose $f: M \rightarrow \mathbb{R}$ is a nonconstant harmonic function and $E$ is an annular end of $M$ of finite type for $f$. Then:

1. If $E$ is conformally $A(0,1)$, then the holomorphic one-form $\omega=d f+i d f^{*}$ 
on $A(0,1)$ extends to a meromorphic one-form $\widetilde{\omega}$ on the closed unit disk $\overline{\mathbb{D}}$ (here $f^{*}$ denotes the locally defined conjugate harmonic function of $f$ ). Furthermore:

(a) If for some $t_{0} \in \mathbb{R}$ the one-complex $f^{-1}\left(t_{0}\right) \cap E$ has $2 k$ ends (note that the number of ends is always even since $f$ alternates values greater and smaller than $t_{0}$ in consecutive components of the complement of $f^{-1}\left(t_{0}\right) \cap E$ around $z=0$ ) and $\widetilde{\omega}$ has a pole at $z=0$, then this pole is of order $k+1$. In particular, for every $t \in \mathbb{R}$, the level set $f^{-1}(t)$ has exactly $2 k$ ends.

(b) $\widetilde{\omega}$ is holomorphic if and only if $f$ is bounded on $E$ (in which case $f$ admits a well-defined harmonic conjugate function on $\overline{\mathbb{D}}$ ).

(c) If $\int_{\alpha}|d f|<\infty$ for some end representative $\alpha$ of $f^{-1}\left(t_{0}\right) \cap E$, then $f$ is bounded on $E$.

2. If $E$ is conformally $A(R, 1)$ for some $R \in(0,1)$, then:

(a) $f$ has angular limits almost everywhere on $\partial_{R}$.

(b) Given $t_{0} \in \mathbb{R}$ such that $f^{-1}\left(t_{0}\right) \cap E$ has a finite number of ends, then the limit set of each end of $f^{-1}\left(t_{0}\right) \cap E$ consists of a single point in $\partial_{R}$. In particular, the closure in $A(R, 1)$ of at least one component of $\left\{z \in A(R, 1) \mid f(z) \neq t_{0}\right\}$ is hyperbolid $\left.\right|^{2}$

In the special case that the flux $\int_{\partial_{1}} \frac{\partial f}{\partial r} d s$ vanishes, then item 2 of Theorem 1.1 follows from the proof of Theorem 7.1 in [6].

Theorem 1.1 is motivated by applications of it to minimal surface theory. For specific applications in [5], we will need the following corollary to Theorem 1.1. We remark that López [4] obtained related results of Picard type for minimal surfaces under the assumption that the second sentence of Corollary 1.2 holds, and that Alarcón and López [1] also applied some of the main results in this paper.

Corollary 1.2 Suppose $X=\left(x_{1}, x_{2}, x_{3}\right): A(R, 1) \rightarrow \mathbb{R}^{3}$ is a proper, conformal minimal immersion and that for some horizontal plane $P \subset \mathbb{R}^{3}$, the one-complex $X^{-1}(P)$ has a finite number $2 k$ of ends. Then $R=0$ and the holomorphic height differential $d x_{3}+i d x_{3}^{*}$ extends meromorphically to $\overline{\mathbb{D}}$ with a pole of order $k+1$. In particular, for every horizontal plane $P^{\prime} \subset \mathbb{R}^{3}$, the level set $X^{-1}\left(P^{\prime}\right)$ has exactly $2 k$ ends.

\footnotetext{
${ }^{2}$ A noncompact Riemann surface $\Sigma$ with boundary is hyperbolic if its boundary fails to have full harmonic measure (equivalently, bounded harmonic functions on $\Sigma$ are not determined by their boundary values); otherwise, $\Sigma$ is called parabolic.
} 
Furthermore, after replacing $A(0,1)$ by $A\left(0, R^{\prime}\right)=\left\{z \in \mathbb{C}|0<| z \mid \leq R^{\prime}\right\}$ for some $R^{\prime} \in(0,1)$, the Gauss map of the induced minimal immersion $\left.X\right|_{A\left(0, R^{\prime}\right)}$ is never vertical. Hence, on $A\left(0, R^{\prime}\right)$, the meromorphic Gauss map of $X$ can be expressed as $g(z)=z^{n} e^{H(z)}$ for some $n \in \mathbb{Z}$ and for some holomorphic function $H: A\left(0, R^{\prime}\right) \rightarrow \mathbb{C}$. Furthermore, the following three statements are equivalent:

1. X has finite total curvature.

2. $H$ is bounded on $A\left(0, R^{\prime}\right)$.

3. There are two nonparallel planes $P_{1}, P_{2}$ such that each of these planes individually intersects the surface transversely in a finite number of immersed curves (if $X$ is not flat, then we can change "there are two" by "for all" in this statement).

Additionally, if the flux $\int_{\alpha} \frac{\partial x_{3}}{\partial \eta} d s$ is finite for some end representative $\alpha$ of $X^{-1}(P)$, then $X$ has finite total curvature and $X(A(R, 1))$ is asymptotic to $P$ with finite multiplicity.

\section{Preliminaries on complex analysis.}

In this section we recall the statements of two classical theorems in the theory of functions of one complex variable that we will apply to prove Theorem 1.1 .

Theorem 2.1 (Plessner [7]) If $F$ is a meromorphic function in the open unit disk $\mathbb{D}$, then, for almost all $\xi \in \partial \mathbb{D}$, either $F$ has a finite angular limit at $\xi$ or $F(S)$ is dense in $\mathbb{C} \cup\{\infty\}$, for every angular sector $S=\{z \in \mathbb{D}|| \arg (1-\bar{z} \xi) \mid<$ $\alpha,|z-\xi|<t\}$ centered at $\xi$ of radius $t$, aperture angle $2 \alpha$ (here $0<\alpha<\frac{\pi}{2}$ ) and median line $[0, \xi]$.

Theorem 2.2 (Privalov $[\overline{8}]$ ) Let $F$ be a meromorphic function in $\mathbb{D}$. If $F$ has angular limit 0 in a subset of positive measure in $\partial \mathbb{D}$, then $F$ vanishes identically.

\section{The proof of Theorem 1.1 .}

Suppose $t_{0} \in \mathbb{R}$ and $f: A(R, 1) \rightarrow \mathbb{R}$ is a nonconstant harmonic function with $f^{-1}\left(t_{0}\right)$ having $n+1$ ends. The ends of $f^{-1}\left(t_{0}\right)$ can be represented by a family $\left\{\alpha_{0}, \alpha_{1}, \ldots, \alpha_{n}\right\}$ of pairwise disjoint proper arcs in $A(R, 1)$. Our first observation in proving Theorem 1.1 is given in the following assertion. 
Assertion 3.1 There exists a simple closed analytic curve $\beta: \mathbb{S}^{1} \rightarrow A(R, 1)$ which is topologically parallel to $\partial_{1}$ and which intersects $f^{-1}\left(t_{0}\right)$ transversely in $n+1$ points $p_{0}=\alpha_{0} \cap \beta, p_{1}=\alpha_{1} \cap \beta, \ldots, p_{n}=\alpha_{n} \cap \beta$. Furthermore, after replacing $A(R, 1)$ by the closure of the subdomain of $A(R, 1)-\beta$ which is disjoint from $\partial_{1}$, then $f^{-1}\left(t_{0}\right)$ consists of $n+1$ disjoint proper arcs representing the ends of $f^{-1}\left(t_{0}\right)$.

Proof. This assertion follows immediately from elementary separation properties of curves and the conformal classification of annular domains.

By Assertion 3.1, after replacing $A(R, 1)$ by a subend and parameterizing this subend by some $A\left(R^{\prime}, 1\right)$, we may assume that $f: A(R, 1) \rightarrow \mathbb{R}$ is harmonic and analytic (up to and including the boundary $\partial_{1}$ ) and $f^{-1}\left(t_{0}\right)$ is a finite collection $\left\{\alpha_{0}, \alpha_{1}, \ldots, \alpha_{n}\right\}$ of pairwise disjoint, properly embedded arcs transverse to $\partial_{1}$, and each arc $\alpha_{i}$ has its starting point $p_{i}$ in $\partial_{1}, i=0,1, \ldots, n$. We can also assume that $\left\{\alpha_{0}, \alpha_{1}, \ldots, \alpha_{n}\right\}$ are cyclically ordered in a counterclockwise manner.

Assertion 3.2 Suppose that $R>0$ and that the limit set $L\left(f^{-1}\left(t_{0}\right)\right) \subset \partial_{R}$ of $f^{-1}\left(t_{0}\right)$ is not equal to $\partial_{R}$. Then, item 2 in the statement of Theorem 1.1 holds.

Proof. Let $\sigma$ be a compact embedded $\operatorname{arc}$ in $\bar{A}(R, 1)-\bigcup_{i=0}^{n} \alpha_{i}$ with one end point in $\partial_{1}$ and the other end point in $\partial_{R}$ (note that $\sigma$ exists since $\partial_{R}-L\left(f^{-1}\left(t_{0}\right)\right) \neq \varnothing$ ). Let $\sigma(\varepsilon)$ be a small, open regular neighborhood of $\sigma$ in $\bar{A}(R, 1)-\bigcup_{i=0}^{n} \alpha_{i}$ which is at a positive distance from $\bigcup_{i=0}^{n} \alpha_{i}$ and chosen so that $\partial[A(R, 1)-\sigma(\varepsilon)]$ is a smooth connected arc. By the conformal classification of annular domains and boundary regularity of holomorphic functions, there exists a conformal diffeomorphism (see Figure 2)

$$
\eta: \Delta=\{z=x+i y \in \mathbb{C}|| z \mid<1, y \geq 0\} \rightarrow[A(R, 1)-\sigma(\varepsilon)] .
$$

Consider the induced harmonic function $\widehat{f}=f \circ \eta: \Delta \rightarrow \mathbb{R}$. Since $\Delta$ is simply connected, $\widehat{f}$ admits a well-defined harmonic conjugate function $\widehat{f}^{*}$. Hence, the function $F=\widehat{f}+i \widehat{f}^{*}: \Delta \rightarrow \mathbb{C}$ is holomorphic. As $f$ does not have critical points in $f^{-1}\left(t_{0}\right), F$ restricted to any of the finite number of components $\eta^{-1}\left(\alpha_{i}\right)$ of $\widehat{f}^{-1}\left(t_{0}\right)(0 \leq i \leq n)$ monotonically parameterizes an interval on the line

$$
L_{t_{0}}=\left\{w=u+i v \in \mathbb{C} \mid u=t_{0}\right\} .
$$

The end points on these intervals on $L_{t_{0}}$ form a finite subset, and thus, it is possible to find a compact interval $\gamma$ in $L_{t_{0}}$ which is disjoint from the end points in the intervals in $F\left(\widehat{f}^{-1}\left(t_{0}\right)\right)$. Therefore, $F^{-1}(\gamma)$ is compact. Hence, after replacing 


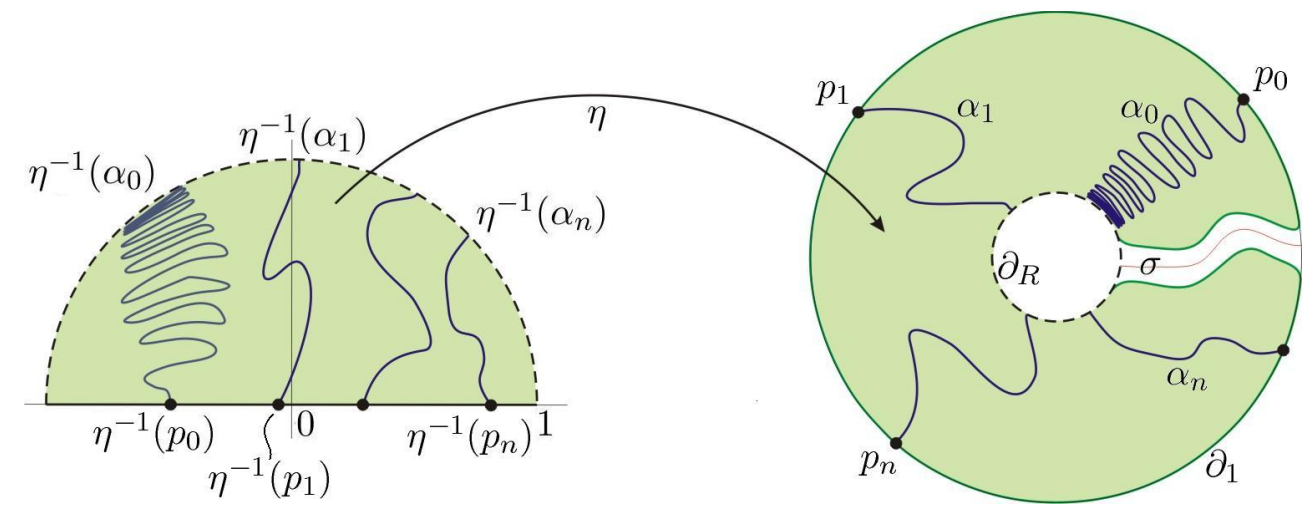

Figure 2: $\eta$ conformally parameterizes the shaded region on the right by a half disk. In fact, we will show that $\alpha_{0}$ with an interval limit set does not occur.

$A(R, 1)$ by a subend, we will assume that $F^{-1}(\gamma)=\emptyset$. Consider the restriction $\left.F\right|_{\operatorname{Int}(\Delta)}: \operatorname{Int}(\Delta) \rightarrow \mathbb{C}-\gamma$ to the interior of $\Delta .\left.F\right|_{\operatorname{Int}(\Delta)}$ is essentially bounded in the sense that its image is contained in a domain conformally equivalent to an open subset of the unit disk via the Riemann mapping theorem. By Plessner's Theorem (Theorem 2.1), $\left.F\right|_{\operatorname{Int}(\Delta)}$ has angular limits almost everywhere on $\left(\mathbb{S}^{1}\right)^{+}=\{z \in$ $\mathbb{C}|| z \mid=1, y \geq 0\}$, and thus, $f$ has angular limits almost everywhere on $\partial_{R}-$ $\sigma(\varepsilon)$. Clearly, by taking smaller and smaller neighborhoods $\sigma(\varepsilon)$, we conclude that item (a) in statement 2 of Theorem 1.1 holds in this case.

We next describe the limit set $L_{i}$ of $\eta^{-1}\left(\alpha_{i}\right), i=0,1, \ldots, n$. Clearly $L_{i} \subset$ $\left(\mathbb{S}^{1}\right)^{+}$. Suppose that for $i$ fixed, $\eta^{-1}\left(\alpha_{i}\right)$ has two distinct limit points $q_{1}, q_{2} \in$ $\left(\mathbb{S}^{1}\right)^{+}$. We claim that in this case, the subarc $I$ of $\left(\mathbb{S}^{1}\right)^{+}$whose extrema are $q_{1}, q_{2}$ is entirely contained in $L_{i}$. Arguing by contradiction, suppose that there exists $s \in \operatorname{Int}(I)$ which is not a limit point of $\eta^{-1}\left(\alpha_{i}\right)$. Then there exists a small $\delta>0$ such that the radial arc $[1-\delta, 1] s \subset \Delta$ is disjoint from $\eta^{-1}\left(\alpha_{i}\right)$. As $\eta^{-1}\left(\alpha_{i}\right)$ is proper in $\Delta$ and disjoint from $[1-\delta, 1] s$, then $\eta^{-1}\left(\alpha_{i}\right)$ eventually lies in the one of the two connected components, say $A$, obtained by removing $[1-\delta, 1] s$ from the $\delta$-neighborhood of $\left(\mathbb{S}^{1}\right)^{+}$in $\Delta$. In particular, $L_{i}$ fails to contain the point in $\left\{q_{1}, q_{2}\right\}$ which does not lie in $\bar{A}$, which is a contradiction. This proves our claim, and therefore, $L_{i}$ is either a compact subarc of $\left(\mathbb{S}^{1}\right)^{+}$or a point.

We claim that all the limit sets $L_{i}$ reduce to points. Suppose on the contrary, that some $L_{i}$ is a subarc of $\left(\mathbb{S}^{1}\right)^{+}$with nonempty interior $\operatorname{Int}\left(L_{i}\right)$. Then almost everywhere on $\operatorname{Int}\left(L_{i}\right)$, the holomorphic function $F$ has angular limits which correspond to the end point $* \in L_{t_{0}} \cup\{\infty\}$ of $F\left(\eta^{-1}\left(\alpha_{i}\right)\right)$. In this case, $F$ would have the constant angular limit $*$ on a set of positive measure of $\left(\mathbb{S}^{1}\right)^{+}$, thereby 
contradicting Privalov's Theorem [8] since $F$ is not constant. This contradiction implies that $L_{i}$ is a single point in $\left(\mathbb{S}^{1}\right)^{+}$for all $i=0,1, \ldots, n$, and so, each end of $f^{-1}\left(t_{0}\right)$ has a unique limit point in $\partial_{R}$. Since we are assuming $R>0$, then the harmonic measure of $\partial_{R}$ is positive, which clearly implies item (b) in statement 2 of Theorem 1.1. Now the proof of Assertion 3.2 is complete.

Recall that the collection $\left\{\alpha_{0}, \alpha_{1}, \ldots, \alpha_{n}\right\}=f^{-1}\left(t_{0}\right)$ of pairwise disjoint arcs is cyclically ordered. For $k=0,1, \ldots, n$, let $D_{k}$ be the union of $\alpha_{k} \cup \alpha_{k+1}$ with the component of $A(R, 1)-f^{-1}\left(t_{0}\right)$ whose boundary contains the $\operatorname{arcs} \alpha_{k}, \alpha_{k+1}$ (in the case of $k=n$ we identify $\alpha_{0}$ with $\alpha_{n+1}$ ). Note that $\partial D_{k}$ is a connected open arc, and $D_{k}$ is simply connected. We will call each $D_{k}$ a (topological) sector of $A(R, 1)$. In the next result we study the conformal character of the sectors $D_{k}$.

Assertion 3.3 Suppose that $R>0$ and that the limit set $L\left(f^{-1}\left(t_{0}\right)\right)$ equals $\partial_{R}$. Then, for each $k=0,1, \ldots, n$ the (topological) sector $D_{k}$ is conformally equivalent to the closed upper halfplane in $\mathbb{C}$ (the conformal diffeomorphism between $D_{k}$ and $\{a+b i \mid b \geq 0\}$ fails to be conformal only at the starting points $p_{k}, p_{k+1}$ of $\left.\alpha_{k}, \alpha_{k+1}\right)$.

Proof. The argument is again by contradiction. Assume that for some $k=0,1, \ldots, n$ the assertion fails. By the Uniformization Theorem and boundary regularity of holomorphic maps, then there exists an bijective map $\phi_{k}: \Delta=\{z=x+i y \in$ $\mathbb{C}|| z \mid<1, y \geq 0\} \rightarrow D_{k}$ which is a conformal immersion except at the two points of $\partial \Delta$ that correspond to the end points $p_{k}, p_{k+1}$ of $\alpha_{k}$ and $\alpha_{k+1}$ in $\partial_{1}$. Since $\phi_{k}$ is a bounded holomorphic function, Theorem 2.1 insures that we can find distinct points $\xi_{1}, \xi_{2} \in\left(\mathbb{S}^{1}\right)^{+}$such that $\phi_{k}$ has an angular limit at $\xi_{i}$, $i=1,2$. Consider a pair of smooth disjoint $\operatorname{arcs} \beta_{1}, \beta_{2} \subset \Delta$, each joining a point of $\phi_{k}^{-1}\left[\left(\partial D_{k} \cap \partial_{1}\right)-\left\{p_{k}, p_{k+1}\right\}\right] \subset \partial \Delta \cap\{y=0\}$ to one of the points $\xi_{1}, \xi_{2}$, and transverse to $\left(\mathbb{S}^{1}\right)^{+}$. Let $D_{k}^{\prime}$ be the subdomain of $\Delta$ whose boundary contains both $\beta_{1}, \beta_{2}$, see Figure 3. Note that the (single) limit point of $\phi_{k}\left(\beta_{1}\right)$ is different from the limit point of $\phi_{k}\left(\beta_{2}\right)$ : otherwise for every point $\xi^{\prime}$ in the open $\operatorname{arc}\left(\mathbb{S}^{1}\right)^{+} \subset \partial \Delta$ with extrema $\xi_{1}, \xi_{2}$, the angular limit of $\phi_{k}$ at $\xi^{\prime}$ exists and is equal to the common limit point of $\phi_{k}\left(\beta_{i}\right), i=1,2$. By Privalov's Theorem (Theorem 2.2, this would lead to a contradiction since $\phi_{k}$ is not constant.

Also note that $\phi_{k}\left(D_{k}^{\prime}\right)$ is a subdomain of $A(R, 1)$ whose boundary consists of $\phi_{k}\left(\beta_{1}\right), \phi_{k}\left(\beta_{2}\right)$, an arc contained in $\partial_{1}$ and an open $\operatorname{arc} \delta \subset \partial_{R}$, and that every point of $\delta$ is a positive distance from the domain $A(R, 1)-D_{k}$. Therefore, $\delta$ is disjoint from the limit set of $f^{-1}\left(t_{0}\right)$, which contradicts one of our hypotheses. Now the proof of the assertion is complete.

We continue with our proof of Theorem 1.1 under the assumption that $R>0$. Let $\Sigma$ be the flat surface with boundary $A(R, 1)-\alpha_{0}$, and let $\bar{\Sigma}$ denote the simply 


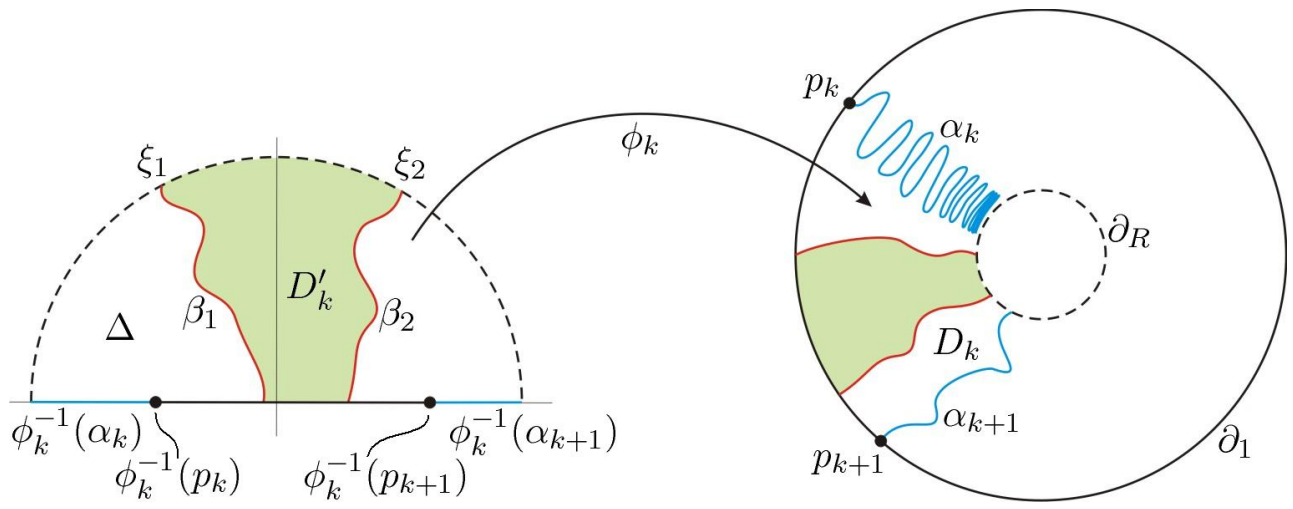

Figure 3: The shaded domain on the right is bounded by curves with distinct limiting end points.

connected flat surface with boundary obtained after attaching to $\Sigma$ two "copies" of $\alpha_{0}$. Note that $\bar{\Sigma}$ has connected boundary, which consists of the arc $\partial_{1}-\alpha_{0}$ together with the two copies of $\alpha_{0}$. For each $k \in\{0,1, \ldots, n\}$, we will consider the "lift" $\widehat{D}_{k}$ of the topological sector $D_{k}$ to $\bar{\Sigma}$. Let $\widehat{f}: \bar{\Sigma} \rightarrow \mathbb{R}$ be the harmonic function given by the "lift" of $f$ to $\bar{\Sigma}$ (clearly $\widehat{f}$ takes equal values at corresponding points in the copies of $\alpha_{0}$ in $\partial \bar{\Sigma}$ ). Since $\bar{\Sigma}$ is simply connected, $\widehat{f}$ admits a well-defined harmonic conjugate function $\widehat{f}^{*}$ on $\bar{\Sigma}$, and so, the function $F=\widehat{f}+i \widehat{f}^{*}: \bar{\Sigma} \rightarrow \mathbb{C}$ is holomorphic.

Note that each sector $\widehat{D}_{k} \subset \bar{\Sigma}$ has its image $F\left(\widehat{D}_{k}\right)$ in one of the two closed halfspaces

$$
\mathbb{C}^{+}\left(t_{0}\right)=\left\{w=u+i v \in \mathbb{C} \mid u \geq t_{0}\right\}, \quad \mathbb{C}^{-}\left(t_{0}\right)=\left\{u+i v \mid u \leq t_{0}\right\} .
$$

Let $L_{t_{0}}=\left\{u+i v \in \mathbb{C} \mid u=t_{0}\right\}$ and note that $F^{-1}\left(L_{t_{0}}\right)$ is the finite ordered set of curves $\left\{\widehat{\alpha}_{0}, \widehat{\alpha}_{1}, \widehat{\alpha}_{2}, \ldots, \widehat{\alpha}_{n}, \widehat{\alpha}_{0}^{\prime}\right\}$ corresponding to the cyclically ordered set of arcs $\left\{\alpha_{0}, \alpha_{1}, \ldots, \alpha_{n}\right\}=f^{-1}\left(t_{0}\right)$; also note that $\partial \widehat{D}_{n}$ contains the arcs $\widehat{\alpha}_{n}$ and $\widehat{\alpha}_{0}^{\prime}$ in its boundary. After reindexing, we can assume that $F\left(\widehat{D}_{k}\right) \subset \mathbb{C}^{+}\left(t_{0}\right)$ for $k$ even and $F\left(\widehat{D}_{k}\right) \subset \mathbb{C}^{-}\left(t_{0}\right)$ for $k$ odd. For $k=0,1, \ldots, n$, let $w_{k} \in L_{t_{0}} \cup\{\infty\}$ be the end point of the half-open $\left.\operatorname{arc} F\right|_{\widehat{\alpha}_{k}}$, and let $w_{n+1} \in L_{t_{0}} \cup\{\infty\}$ be the corresponding end point of $\left.F\right|_{\widehat{\alpha}_{0}^{\prime}}$.

Assertion 3.4 If $R>0$, then item 2 of Theorem 1.1 holds.

Proof. By Assertion 3.2, we only need to arrive to a contradiction provided that $L\left(f^{-1}\left(t_{0}\right)\right)=\partial_{R}$. So assume $L\left(f^{-1}\left(t_{0}\right)\right)=\partial_{R}$. We first check that with the 
notation above, then $w_{k}=w_{k+1}$ for all $k=0,1, \ldots, n$. Consider the restriction $\left.F\right|_{\widehat{D}_{k}}$, which is a holomorphic function whose image is contained in, say, $\mathbb{C}^{+}\left(t_{0}\right)$. Since we are assuming $L\left(f^{-1}\left(t_{0}\right)\right)=\partial_{R}$, Assertion 3.3 insures that there exists a bijective map $\psi_{k}:\{a+i b \in \mathbb{C} \mid b \geq 0\} \rightarrow \widehat{D}_{k}$ which is conformal everywhere on the closed upper halfplane except at the two points $q_{k}, q_{k+1}$ in $\{b=0\}$ which correspond respectively to the starting points $\widehat{p}_{k}, \widehat{p}_{k+1}$ of $\widehat{\alpha}_{k}, \widehat{\alpha}_{k+1}$, respectively. Schwartz's reflection principle applied to the restriction of $F \circ \psi_{k}$ to $\{a+i b \mid$ $b \geq 0\}-\left[q_{k}, q_{k+1}\right]$ (here $\left[q_{k}, q_{k+1}\right]$ denotes the closed interval in the real axis with extrema $\left.q_{k}, q_{k+1}\right)$, and produces a holomorphic function $\widetilde{F}_{k}: \mathbb{C}-\left[q_{k}, q_{k+1}\right] \rightarrow \mathbb{C}$ such that

- $\widetilde{F}_{k}$ extends continuously to the metric completion $\mathcal{C}$ of $\mathbb{C}-\left[q_{k}, q_{k+1}\right]$ (note that $\mathcal{C}$ is conformally $A(0,1)$ ). We denote these extensions by the same symbols $\widetilde{F}_{k}$.

- $\widetilde{F}_{k}$ maps $\mathbb{R}-\left[q_{k}, q_{k+1}\right]$ into the line $L_{t_{0}}$ and maps each of the two copies of the interval $\left[q_{k}, q_{k+1}\right]$, when considered inside the boundary of $\mathcal{C}$, into the union of $F\left(\partial \widehat{D}_{k} \cap \partial_{1}\right)$ and its reflected image with respect to $L_{t_{0}}$. Furthermore, the preimage by $\widetilde{F}_{k}$ of every point in $L_{t_{0}}$ consists of at most two points in the real line $\{b=0\}$, and for some point in $L_{t_{0}}$, its preimage by $\widetilde{F}_{k}$ consists of at most one point.

By Picard's theorem, $\widetilde{F}_{k}$ extends meromorphically across $\infty$, and its extension is a conformal diffeomorphism from a neighborhood $U_{k}$ of $\infty$ in $\mathbb{C} \cup\{\infty\}$ into its image. In particular, the limit point $w_{k}$ of $\widetilde{F}_{k}\left(\psi_{k}^{-1}\left(\widehat{\alpha}_{k}\right)\right)$ equals the limit point $w_{k+1}$ of $\widetilde{F}_{k}\left(\psi_{k}^{-1}\left(\widehat{\alpha}_{k+1}\right)\right)$, as desired.

We now consider the special case where $w_{0}=\infty$. In this case, the pullback by $\left.\widetilde{F}_{k}\right|_{U_{k}}$ of the complete flat metric $|d w|^{2}$ is a complete flat metric on $U_{k}$. Furthermore, for each $k$ the equality $F^{*}|d w|^{2}=\left(\psi_{k}^{-1}\right)^{*}\left(\left.\widetilde{F}_{k}\right|_{U_{k} \cap\{b \geq 0\}}\right)^{*}|d w|^{2}$ holds on some end representative $E_{k}$ of $D_{k}$. Therefore $F$ induces under pullback a complete flat metric on $\cup_{k=0}^{n} E_{k}$, which is an end representative of $\bar{\Sigma}$. Clearly this complete flat metric on this end representative of $\bar{\Sigma}$ descends to a complete flat metric on an end representative of $A(R, 1)$ when $w_{0}=\infty$. This contradicts the assumption that $R$ is positive (because any such complete flat annulus has quadratic area growth by a direct application of the Gauss-Bonnet formula together with the first and second variation of area formulas, and the fact that annular ends with at most quadratic area growth are parabolic, see [3]).

On the other hand if $w_{0}$ is finite, then the arguments in the last paragraph apply to the holomorphic function $\frac{1}{\widetilde{F}_{k}-w_{0}}$ and lead to a similar contradiction. This finishes the proof of Assertion 3.4 
In order to complete the proof of Theorem 1.1 it remains to demonstrate item 1 of the theorem. So from now on suppose $R=0$. As we did just after Assertion 3.1. we can assume that $f: A(0,1) \rightarrow \mathbb{R}$ is harmonic and analytic (up to and including the boundary $\left.\partial_{1}\right)$ and $f^{-1}\left(t_{0}\right)$ is a cyclically ordered finite collection $\left\{\alpha_{0}, \alpha_{1}, \ldots, \alpha_{n}\right\}$ of pairwise disjoint, properly embedded arcs transverse to $\partial_{1}$, and each arc has its starting point in $\partial_{1}$ and limits to $z=0$. Also the arguments just before Assertion 3.3 lead us to define the topological sectors $D_{k}, k=0,1, \ldots, n$, each one being the union of $\alpha_{k} \cup \alpha_{k+1}$ with the component of $A(0,1)-f^{-1}\left(t_{0}\right)$ whose boundary contains the arcs $\alpha_{k}, \alpha_{k+1}$ (with $\alpha_{0}=\alpha_{n+1}$ if $k=n$ ), and with an arc in $\partial_{1}$. Note that these sectors $D_{k}$ are still parabolic in our new setting of $R=0$, since the conformal structure of the annulus $A(0,1)$ is parabolic and the sectors $D_{k}$ are then proper subdomains of a parabolic surface.

Repeating the arguments before Assertion 3.4, we cut $A(0,1)$ along $\alpha_{0}$ and then reattach the cutting curve twice to obtain a simply connected surface $\bar{\Sigma}$, a holomorphic function $F=\widehat{f}+i \widehat{f}^{*}: \bar{\Sigma} \rightarrow \mathbb{C}$ and a finite, ordered set of arcs $\left\{\widehat{\alpha}_{0}, \widehat{\alpha}_{1}, \widehat{\alpha}_{2}, \ldots, \widehat{\alpha}_{n}, \widehat{\alpha}_{0}^{\prime}\right\}$ which correspond to $\left\{\alpha_{0}, \alpha_{1}, \ldots, \alpha_{n}\right\}=f^{-1}\left(t_{0}\right) \subset$ $A(0,1)$. By the arguments in the proof of Assertion 3.4 the holomorphic differential $d F$ on $\bar{\Sigma}$ descends to the holomorphic differential $\omega=d f+i d f^{*}$ on $A(0,1)$, which extends to a meromorphic differential $\widetilde{\omega}$ on $\overline{\mathbb{D}}=\bar{A}(0,1)$.

In order to prove item 1 (a), note that if $\widetilde{\omega}$ is holomorphic at $0 \in \bar{A}(0,1)$, then $d \widehat{F}$ is holomorphic at $\infty$ (with the same notation as in the proof of Assertion 3.4). This implies that $F$ is holomorphic at 0 and so, $f$ is bounded on $E$. Reciprocally, if $f$ is bounded then $F$ is bounded as well, which implies $\widetilde{\omega}=d F$ is holomorphic.

Finally we prove item 1(b) of Theorem 1.1. Since length $(\alpha)=\int_{\alpha}|* d f|=$ $\int_{\alpha}|d f|<\infty$, then the common limit point $w_{0}=\ldots=w_{n}=w_{n+1}$ corresponds a finite point. In this case, the arguments in the last paragraph demonstrate that $f$ is bounded.

Remark 3.5 Suppose $R>0$ and $f: A(R, 1) \rightarrow \mathbb{R}$ is a nonconstant harmonic function with angular limits almost everywhere on $\partial_{R}$. Then the arguments in the proof of Theorem 1.1 can also be applied to demonstrate the following result: For every $t \in \mathbb{R}$, each proper arc (piecewise smooth) in the boundary of a component of $\{z \in A(1, R) \mid f(z) \geq t\}$ or of $\{z \in A(1, R) \mid f(z) \leq t\}$ has a unique limit point in $\partial_{R}$. In particular, each nonlimit end of the 1-complex $f^{-1}(t)$ has a unique limit point in $\partial_{R}$.

\section{The proof of Corollary 1.2 .}

In this section, we will apply the following theorem of Collin, Kusner, Meeks and Rosenberg [2] to prove Corollary 1.2 
Theorem 4.1 If $X: \Sigma \rightarrow \mathbb{R}^{3}$ is a properly immersed minimal surface with boundary (possibly empty) which is contained in a halfspace of $\mathbb{R}^{3}$, then $\Sigma$ is parabolic.

Let $R \in[0,1)$. Suppose $X: A(R, 1) \rightarrow \mathbb{R}^{3}$ is a conformal, proper minimal immersion such that $X^{-1}(P)$ has a finite number of ends for some horizontal plane $P \subset \mathbb{R}^{3}$ at height $t_{0} \in \mathbb{R}$.

We claim that $R=0$. Otherwise $R>0, A(R, 1)$ is an annular end of finite type for $x_{3}$ and some component of $x_{3}^{-1}\left(\left(-\infty, t_{0}\right]\right)$ or $x_{3}^{-1}\left(\left[t_{0}, \infty\right)\right)$ is hyperbolic by item 2 of Theorem 1.1. But such a component must be parabolic by Theorem 4.1. since $X$ restricted to this component is a properly immersed minimal surface contained in a halfspace of $\mathbb{R}^{3}$. Hence $R=0$.

By item 1 of Theorem 1.1, the holomorphic one-form $d x_{3}+i d x_{3}^{*}$ extends to a meromorphic one-form on $\overline{\mathbb{D}}=\bar{A}(0,1)$. By regularity of the induced metric, the meromorphic Gauss map $g: A(0,1) \rightarrow \mathbb{C} \cup\{\infty\}$ of $X$ achieves the values $0, \infty$, corresponding to the north and south poles of $\mathbb{S}^{2}$ and equal to the unit normals of $P$, only a finite number of times. Hence, $g$ misses $0, \infty$ on $A\left(0, R^{\prime}\right)$ for some $R^{\prime} \in(0,1]$. For some $k \in \mathbb{Z}, z^{-k} g$ induces the zero map from $\pi_{1}\left(A\left(0, R^{\prime}\right)\right)$ to $\pi_{1}(\mathbb{C}-\{0\})$ and thus, by elementary covering space theory, $z^{-k} g(z)=e^{H(z)}$ for some holomorphic function $H$ on $A\left(0, R^{\prime}\right)$. This completes the proof of the main statement of Corollary 1.2 .

We next prove the equivalence between items $1-3$ of Corollary 1.2 . The only implication which is not obvious is that 3 implies 1 , so assume 3 holds. The main statement of Corollary 1.2 applied to each of the planes $P_{1}, P_{2}$ implies that on some end $A_{R_{1}}=\left\{z \in A(0,1)|0<| z \mid \leq R_{1}<1\right\}$ of $A(0,1)$, the Gauss map $g$ misses the four values corresponding to the two pairs of antipodal points of $\mathbb{S}^{2}$ which are orthogonal to $P_{1}$ or $P_{2}$. Since $A_{R_{1}}$ is conformally a punctured disk, Picard's theorem can be applied to $g$ and gives that $g$ extends across the puncture to a meromorphic function on $\overline{\mathbb{D}}=\overline{A(0,1)}$. Hence, $X$ has finite total curvature.

Finally suppose that $\int_{\alpha} \frac{\partial x_{3}}{\partial \eta} d s$ is finite for some end representative of $X_{3}^{-1}(P)$. Then item 1.(b) of Theorem 1.1 implies that $x_{3}$ is bounded on the minimal end $E=X(A(0,1))$. In particular, $E$ is contained in a horizontal slab. In this setting, Lemma 2.2 in [2] insures that $E$ has quadratic area growth. Since the Gaussian curvature of $E$ is nonpositive, a standard application of the first and second variation formulas for area imply that $E$ has finite total curvature. In this situation, it is well-known that $E$ is asymptotic to $P$ with finite multiplicity. Now Corollary 1.2 is proved. 
William H. Meeks, III at profmeeks@gmail.com

Mathematics Department, University of Massachusetts, Amherst, MA 01003

Joaquín Pérez at jperez@ugr.es

Department of Geometry and Topology and Institute of Mathematics

(IEMath-GR), University of Granada, 18071, Granada, Spain

\section{References}

[1] A. Alarcón and F. J. López. On harmonic quasiconformal immersions in $\mathbb{R}^{3}$. Transactions of the AMS, 365(4):1711-1742, 2013. MR3009644, Zbl 1320.53076 .

[2] P. Collin, R. Kusner, W. H. Meeks III, and H. Rosenberg. The geometry, conformal structure and topology of minimal surfaces with infinite topology. $J$. Differential Geom., 67:377-393, 2004. MR2153082, Zbl 1098.53006.

[3] A. Grigor'yan. Analytic and geometric background of recurrence and nonexplosion of Brownian motion on Riemannian manifolds. Bull. of A.M.S, 36(2):135-249, 1999. MR1659871, Zbl 0927.58019.

[4] F. J. López. Some Picard theorems for minimal surfaces. Trans. Amer. Math. Soc., 356(2):703-733, 2004. MR2022717 (2004j:53017), Zbl 1121.53007.

[5] W. H. Meeks III and J. Pérez. Embedded minimal surfaces of finite topology. Preprint at http://arxiv.org/pdf/1506.07793v1.pdf.

[6] W. H. Meeks III and H. Rosenberg. Maximum principles at infinity. J. Differential Geom., 79(1):141-165, 2008. MR2401421, Zbl pre05285650.

[7] A. I. Plessner. Über das verhalten analytischer functionen am rande ihres definitionbereichs. J. Reine Angew. Math., 158:219-227, 1927. JFM 53.0284.01.

[8] I. I. Privalov. Randeigenschaften analytischer Funktionen. 2. Überarb. und erg. Aufl. Hochschulbücher für Mathematik. 25. Berlin: VEB Deutscher Verlag der Wissenschaften VIII, 247 S. mit 10 Abb., 1956. Zbl 0073.06501. 